Observati on of Enhanced Radi al Transport of Energet i c I on due to Energet i c Parti cle Node Dest abi I i zed by Hel i cal I y- $t$ r apped Energet i c I on in the Large Hel i cal Devi ce

\begin{tabular}{|l|l|}
\hline $\begin{array}{l}\text { j our nal or } \\
\text { publ i cat } \mathrm{i} \text { on } \mathrm{t} \text { i } \mathrm{tl} \text { e }\end{array}$ & Nucl ear Fusi on \\
\hline vol une & 58 \\
\hline number & 4 \\
\hline page $\mathrm{r}$ ange & 044001 \\
\hline year & $2018-02-13$ \\
\hline URL & ht t p: //hdl . handl e. net /10655/00012565 \\
\hline
\end{tabular}


Observation of Enhanced Radial Transport of Energetic Ion due to Energetic Particle Mode Destabilized by Helically-trapped Energetic Ion in the Large Helical Device

K. Ogawa ${ }^{1,2}$, M. Isobe ${ }^{1,2}$, H. Kawase ${ }^{2}$, T. Nishitani ${ }^{1}$, R. Seki ${ }^{1,2}$, M. Osakabe ${ }^{1,2}$, and LHD Experiment Group ${ }^{1}$

1) National Institute for Fusion Science, National Institutes of Natural Sciences, Toki-city, Gifu, Japan

2) SOKENDAI (The Graduate University for Advanced Studies), Toki-city, Gifu, Japan

Deuterium experiment was initiated to achieve higher-temperature and higher-density plasmas in March 2017 in the Large Helical Device (LHD). The central ion temperature notably increases compared with that in hydrogen experiments. However, energeticparticle modes called the helically-trapped energetic-ion-driven resistive interchange modes (EIC) are often excited by intensive perpendicular neutral beam injections on high ion-temperature discharges. The mode leads to significant decrease of the ion temperature or to limiting the sustainment of the high ion-temperature state. To understand the effect of EIC on the energetic-ion confinement, the radial transport of energetic ions is studied by means of the neutron flux monitor and vertical neutron camera newly installed on the LHD. Decreases of line-integrated neutron profile in core channels show that helicallytrapped energetic ions are lost from the plasma.

Introduction

Sustainment of high-temperature and high-density plasmas is required for realizing the nuclear fusion reactor. However, energetic-particle transport induced by magnetohydrodynamics (MHD) modes triggered by energetic particles may lead to prevention of achieving high-performance plasmas and/or sustaining long-duration of high-performance discharges. In currently performed neutral-beam-heated deuterium plasma discharges, neutrons are mainly created by beam-plasma reactions. This means that neutron diagnostics can be used for studying energetic-ion physics. In tokamaks, the energetic-ion-driven off-axis-fishbone-like mode triggers a resistive wall mode (RWM) in high plasma pressure discharges in DIII-D and JT-60U $[1,2]$. Due to the RWM, the peak neutron rate decreases by $12 \%$ within one millisecond which suggests that energetic ions are immediately lost due to the RWM. In the Large Helical Device (LHD), deuterium experiment was initiated to achieve higher-temperature and higher-density plasmas in March 2017 [3]. The central ion temperature reaches around $10 \mathrm{keV}$ using neutral beam 
injections. However, the resistive interchange modes destabilized by energetic particles called the helically-trapped energetic-ion-driven resistive interchange mode (EIC) [4] are often excited by intensive perpendicular neutral beam injections. Accordingly, sustainment of the high ion-temperature state was limited. Hence, to understand the effect of the EIC on the beam ion confinement, beam ion transport and/or beam ion loss are studied by means of the comprehensive neutron diagnostics newly installed on the LHD such as a neutron flux monitor (NFM) and vertical neutron camera (VNC). This paper shows the observation of radial transport of beam ions due to the EIC.

\section{Experimental Setups}

The LHD is the world's largest heliotron device with a major radius/minor radius of 3.9 $\mathrm{m} / \sim 0.6 \mathrm{~m}$. The maximum toroidal magnetic field strength on the axis position is around $3 \mathrm{~T}$. The LHD is equipped with three tangentially-injected beams based on a negative-ion source (NNB) having acceleration voltage of around $180 \mathrm{keV}$ and two perpendicularlyinjected beams (PNB) based on a positive-ion source having acceleration voltage of 40 to $80 \mathrm{keV}$. The total injection power of NNB is around $15 \mathrm{MW}$, whereas that of PNB is around $18 \mathrm{MW}$. The typical pitch angle of beam ions injected by NNB is around 30 degrees, whereas that of PNB is 90 degrees. Therefore, beam ions injected by NNB mainly have passing orbits and those injected by PNB mainly have helically-trapped orbits. The total neutron emission rate $\left(S_{\mathrm{n}}\right)$ is measured by the absolutely calibrated NFM. The NFM consists of three ${ }^{235} \mathrm{U}$ fission chambers, one ${ }^{10} \mathrm{~B}$ proportional counter, and two ${ }^{3} \mathrm{He}$ proportional counters. The primary detector for measuring $S_{\mathrm{n}}$ is installed on the central axis of the LHD. The features of the NFM are wide dynamic range (around ten orders of magnitude) and fast response (time response of $2 \mathrm{~ms}$ ) which are realized by the digital signal processing unit equipped with a leading-edge field programmable gate array (FPGA). The details of NFM are discussed in Ref. 5. Line-integrated neutron profiles are measured by using the VNC newly installed on the basement level of the torus hall (Fig.1)

[6]. The VNC capable of operation over MHz counting rate consists of an eleven-channel collimator made from a heavy concrete, stilbene fast-neutron scintillation detectors coupled with a photomultiplier having a high-gain stability, and the data acquisition system (DAQ) equipped with the leading-edge fast analogue to digital converter and FPGA. The waveform acquired with $1 \mathrm{GHz}$ sampling rate and shaping parameters of each pulse can be obtained simultaneously. Therefore, online and offline neutron-gamma discrimination are possible. The line-integrated neutron profiles were measured in three different magnetic axis conditions with deuterium NNB injection on line-averaged electron density $n_{\mathrm{e} \_ \text {av }}$ of around $1.0 \times 10^{19} \mathrm{~m}^{-3}$ (Fig. 2). In these experiments, the plasma 
center position $R_{\mathrm{ax}}$ obtained by the electron temperature profile measured by Thomson scattering diagnostics were $3.68 \mathrm{~m}, 3.82 \mathrm{~m}$, and $3.95 \mathrm{~m}$, respectively. It shows that the peak of line-integrated neutron profile shifted outward according to $R_{\text {ax. }}$. We demonstrates that the VNC works successfully to measure neutron emission profile of LHD plasmas.

\section{Experimental Results}

Figure 3 shows the typical time evolution of high ion-temperature discharges in the LHD. Here, $R_{\text {ax } \_ \text {vac, }} B t, P_{\mathrm{NB}}, P_{\mathrm{rad}}, T_{\mathrm{e} 0}, T_{\mathrm{i} 0}$, and $b_{\theta}$ represent the magnetic axis position in vacuum, the toroidal magnetic field strength, injection powers of NBs, the radiation power measured by the resistive bolometer [7], the central electron temperature measured by the Thomson scattering diagnostics [8], the central ion temperature measured by the charge exchange recombination spectroscopy diagnostics [9], and the magnetic fluctuation amplitude of EIC derived from the signal of Mirnov coil installed on the vacuum vessel [10] by using numerical band-pass filter of $1 \mathrm{kHz}$ to $200 \mathrm{kHz}$. Here, the range of $n_{\mathrm{e} \_}$av and $T_{\mathrm{e} 0}$ is $(0.5 \sim 2.0) \times 10^{19} \mathrm{~m}^{-3}$ and $1 \sim 4 \mathrm{keV}$, respectively. Note that the line-integrated electron density rapidly increases due to the carbon pellet injection at $t$ of $4.52 \mathrm{~s}$ in order to obtain sufficient intensity of the carbon line emission to measure the ion temperature. In this experiment, NNBs inject hydrogen beams whereas PNBs inject deuterium beams. Strong bursting EICs are clearly seen by the intensive PNB injection in $b_{\theta}$. EIC bursts cause concurrent decreases of $S_{\mathrm{n}}$, increases of $P_{\mathrm{rad}}$, and decreases of $T_{\mathrm{i} 0}$. This suggests that beam ions injected perpendicularly are lost due to EIC bursts from the plasma and hit the plasma facing components. Here, decreases of $T_{\mathrm{i} 0}$ is the result of degradation of beam ion confinement by EIC burst. It is worth noting that almost $50 \%$ of $S_{\mathrm{n}}$ is dropped within 10 ms. Line-integrated neutron profiles are measured by the VNC in this discharge. The time evolutions of square root of $b_{\theta}^{2}$ together with the line-integrated neutron profile from 4.5 $\mathrm{s}$ to $5.5 \mathrm{~s}$ are shown in Fig. 4. Here, the time bin of neutron counts $\left(C_{\mathrm{n}}\right)$ is set to be $10 \mathrm{~ms}$. We see the change of the line-integrated profile due to EIC busts. Here, the maximum pulse counting rate is around $4 \times 10^{4} \mathrm{cps}$ because the neutron-gamma ratio is 1 to 3 in the central channel, i.e., $R$ of $3.72 \mathrm{~m}$. Figure 5 shows time evolutions of $P_{\mathrm{NB}}$ of $\mathrm{PNB}, b_{\theta}$ and $C_{\mathrm{n}}$ in eight channels. Here, $R$ of $3.72 \mathrm{~m}, 3.90 \mathrm{~m}$, and $4.17 \mathrm{~m}$ correspond to the distance between plasma axis and VNC sight line normalized by plasma minor radius $\left(r_{\mathrm{min}} / a\right)$ of $0.0,0.5$, and that of 1.0, respectively. Figure 5 clearly shows that intermittent drops of line-integrated neutron counts in the plasma core channel synchronized with EIC bursts. Neutron counts decay gradually without EIC burst from $t$ of $5.16 \mathrm{~s}$; the e-folding decay time of $C_{\mathrm{n}}$ on $R$ of $3.72 \mathrm{~m}$ is around $50 \mathrm{~ms}$ which is almost the same as the decay time of $S$ n. On the other hand, correlated with EIC burst, neutron counts decay rapidly, and the 
decay times becomes significantly short. Here, $n_{\mathrm{e} \_ \text {avg }}$ and $T_{\mathrm{e} 0}$ are not significantly changed to change the slowing down time of beam ions considerably in the time period as shown in Fig. 3. Figure 6 a) shows the $C_{\mathrm{n}}$ profiles before and after EIC burst. The figure shows that in the core channel ( $r_{\min } / a$ less than 0.5$)$ the $C_{\mathrm{n}}$ drops up to $50 \%$, whereas that in the edge channel stays almost the same. Figure $6 \mathrm{~b}$ ) shows the sight line of VNC and the typical density profile of helically-trapped beam ions created by PNB injections [11]. The helically-trapped beam ion density profile shows that it has a peak around $r / a$ of 0.8 where EIC excites. Relatively narrow neutron profile obtained in Fig. 6 a) is consistent with that expected from a density of helically-trapped beam ion. In this experiment, the e-folding decay time of $S_{\mathrm{n}}\left(\tau_{\mathrm{n}}\right)$, which scales almost linearly with Spizter slowing down time parameter, i.e. $T_{\mathrm{e}}^{3 / 2} / n_{\mathrm{e}}$ [12] becomes 0.2 times smaller i.e. from $50 \mathrm{~ms}$ to $10 \mathrm{~ms}$ due to EIC bursts. If beam ions existing around $r / a$ of 0.8 are transported to the last closed flux surface (LCFS), $\tau_{\mathrm{n}}$ becomes 0.27 times smaller because the ratio of $T \mathrm{e}^{3 / 2} / n_{\mathrm{e}}$ at the LCFS to $T_{\mathrm{e}}^{3 / 2} / n_{\mathrm{e}}$ at $r / a$ of 0.8 is 0.27 . Here, $T_{\mathrm{e}} / n_{\mathrm{e}}$ at $r / a$ of 0.8 and at the LCFS are 1.5 $\mathrm{keV} / 1.4 \times 10^{19} \mathrm{~m}^{-3}$, and $0.37 \mathrm{keV} / 0.58 \times 10^{19} \mathrm{~m}^{-3}$, respectively. It means that significant change of $\tau_{\mathrm{n}}$ due to EIC is due to beam ion losses. Therefore, a decrease of $C_{\mathrm{n}}$ in central channels can be interpreted by loss/expulsion of helically-trapped beam ions due to EIC bursts.

\section{Summary}

Energetic-particle modes named EICs destabilized by intensive PNB injections are often excited on high ion-temperature discharges in the LHD. Intermittent decreases of $S_{\mathrm{n}}$ up to $50 \%$ associated with EIC are observed using the NFM. The line-integrated neutron profile measured by the VNC remarkably changes due to EIC bursts. Sufficient drops of $C_{\mathrm{n}}$ are caused in the core channels $\left(r_{\min } / a\right.$ less than 0.5$)$, whereas there is almost no change of $C_{\mathrm{n}}$ in the edge channels. This shows that helically-trapped beam ions are transported and lost from the plasma by the EIC.

\section{Acknowledgments}

This work is supported partly by LHD project budgets (ULGG801, ULHH003, and ULHH034).

\section{References}

[1] Okabayashi M. et al 2011 Phys. Plasmas 18056112.

[2] Matsunaga G. et al 2010 Nucl. Fusion 50084003.

[3] Osakabe M. et al 2017 Fusion Sci. Technol. 72199. 
[4] Du X. D. et al 2015 Nucl. Fusion 56016002.

[5] Isobe M. et al 2014 Rev. Sci. Instrum 85 11E114.

[6] Ogawa K. et al 2014 Rev. Sci. Instrum 85 11E110.

[7] Peterson B. J. et al 2010 Fusion Sci. Technol. 58412.

[8] Yamada I. et al 2010 Fusion Sci. Technol. 58345.

[9] Yoshinuma M. et al 2010 Fusion Sci. Technol. 58375.

[10] Sakakibara S. et al 2010 Fusion Sci. Technol. 58471.

[11] Seki R. et al 2010 Plasma Fusion Res. 5014.

[12] Strachan J. et al 1981 Nuclear Fusion 2167. 


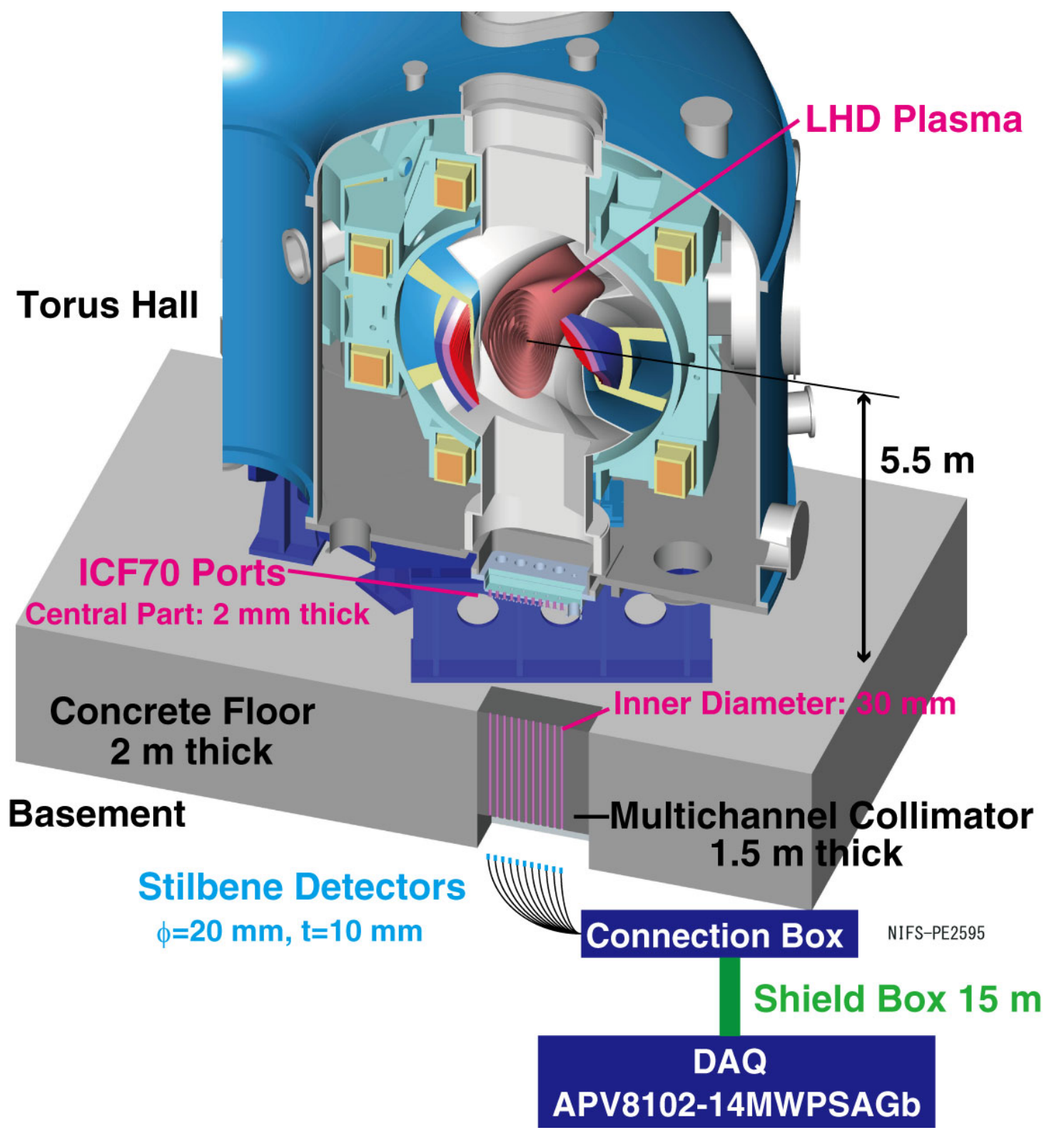

Fig. 1 Schematic drawing of vertical neutron camera installed on the LHD. 


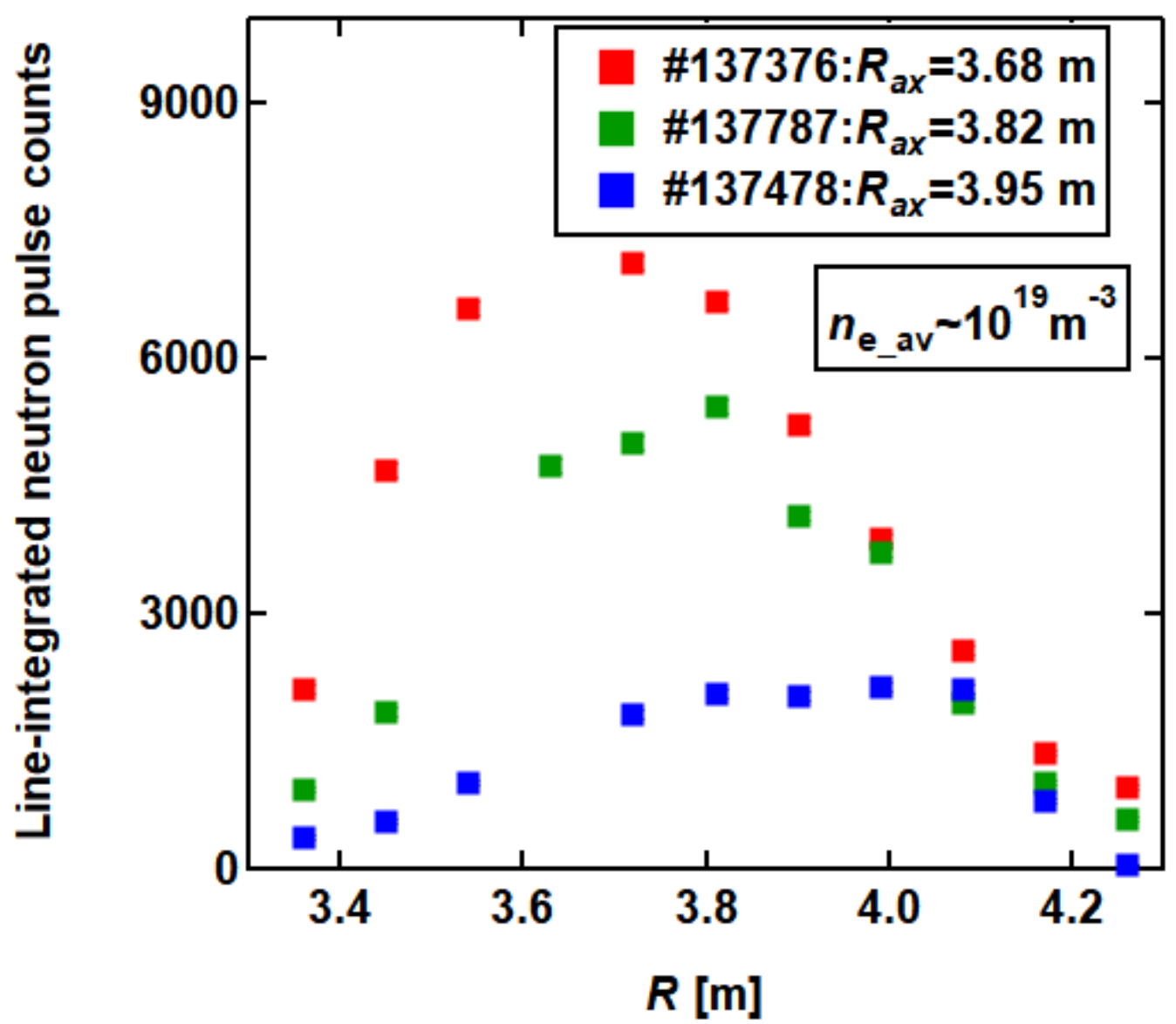

Fig. 2 Line-integrated radial neutron profile obtained in various plasma axis conditions with deuterium NNB injection. The peak of the line-integrated neutron profile changed according to plasma axis position. 


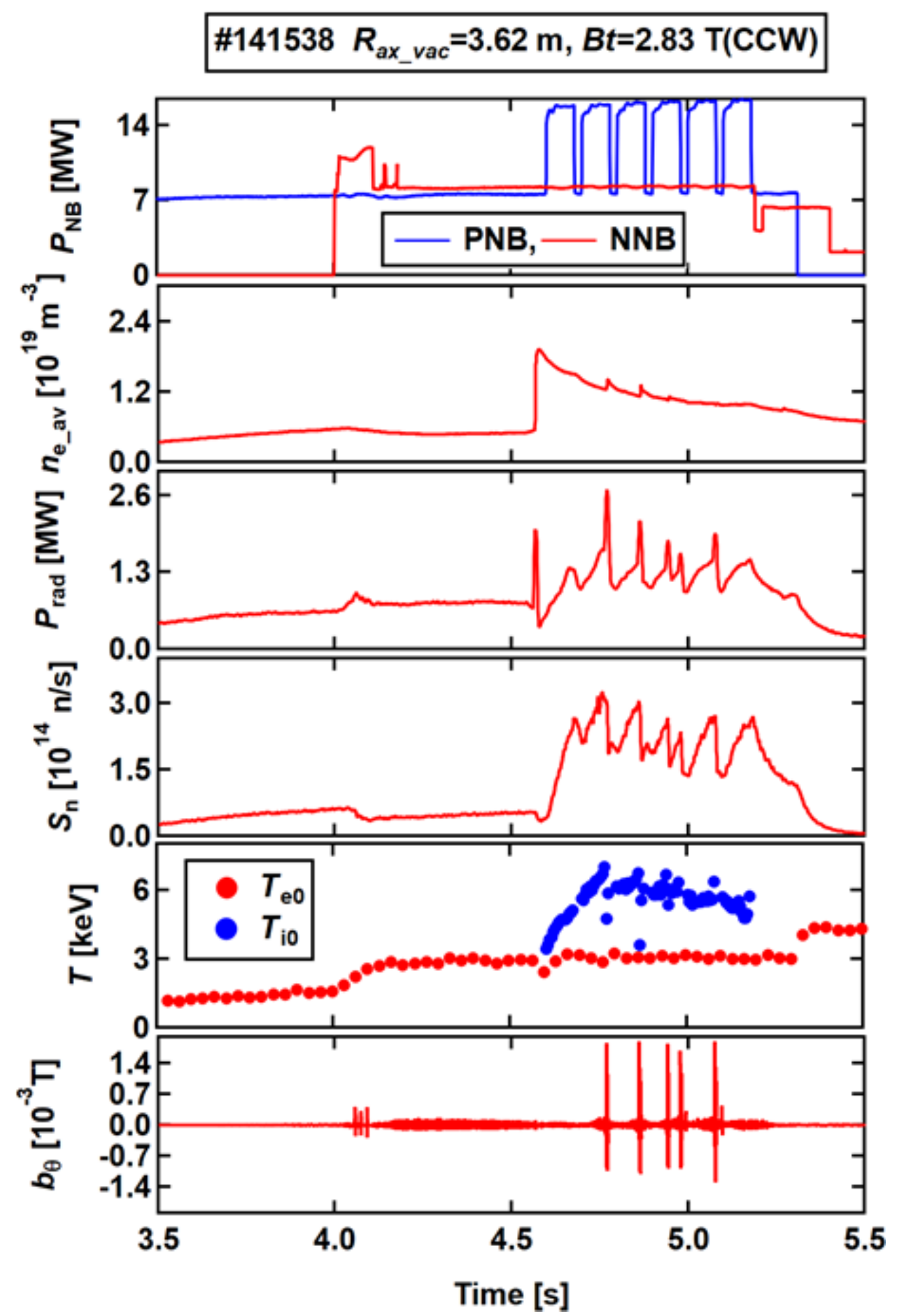

Fig. 3 Typical time evolution of plasma discharges in $R_{\text {ax_vac }} / B t$ of $3.62 \mathrm{~m} / 2.83 \mathrm{~T}$. Intermittent MHD mode bursts are observed with intensive perpendicular neutral beam injection. 


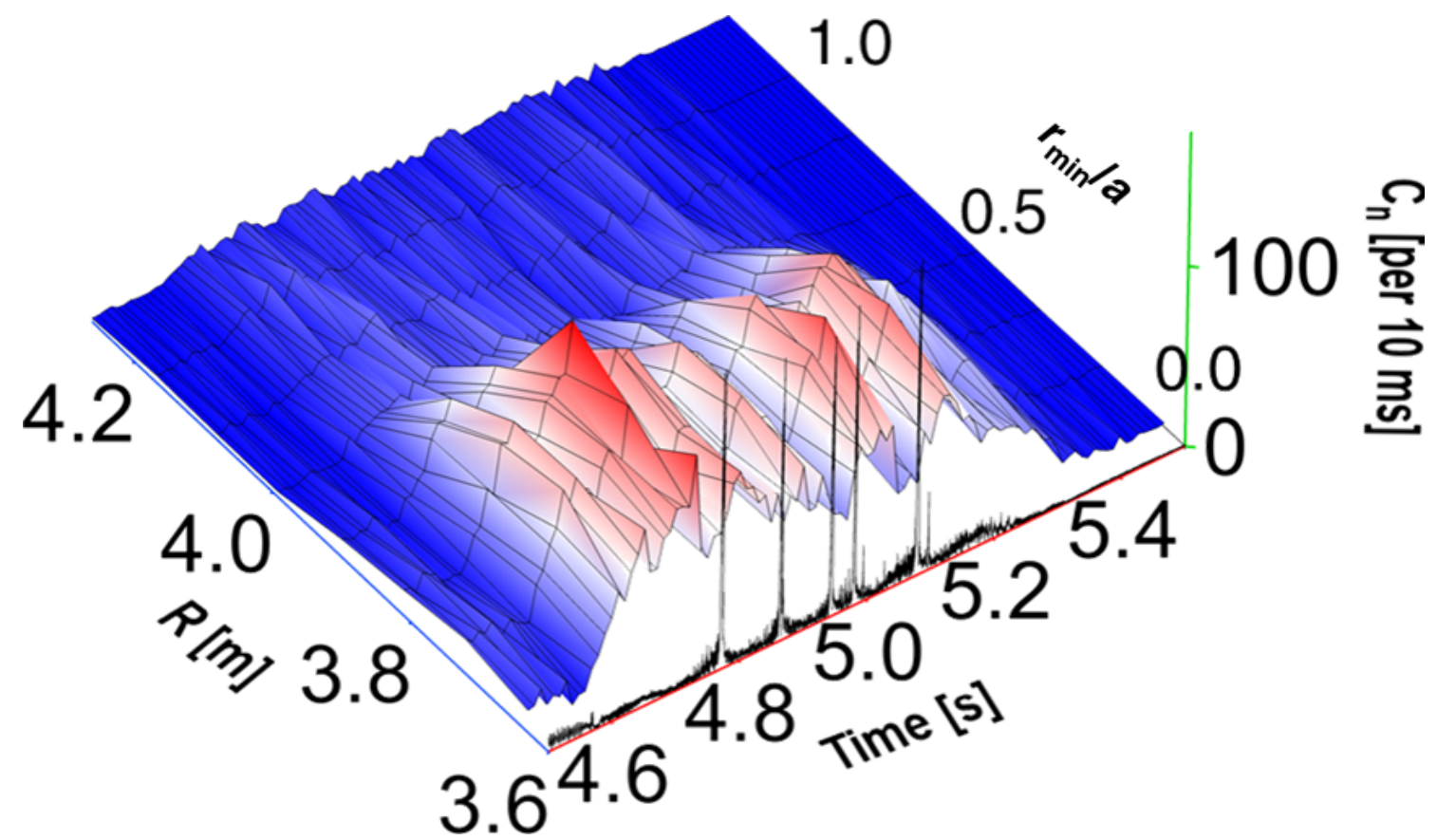

Fig. 4 Time evolutions of magnetic fluctuation amplitude (black line) and line-integrated neutron profile (\#141538). Change of line-integrated neutron profile synchronized with EIC bursts are observed. 


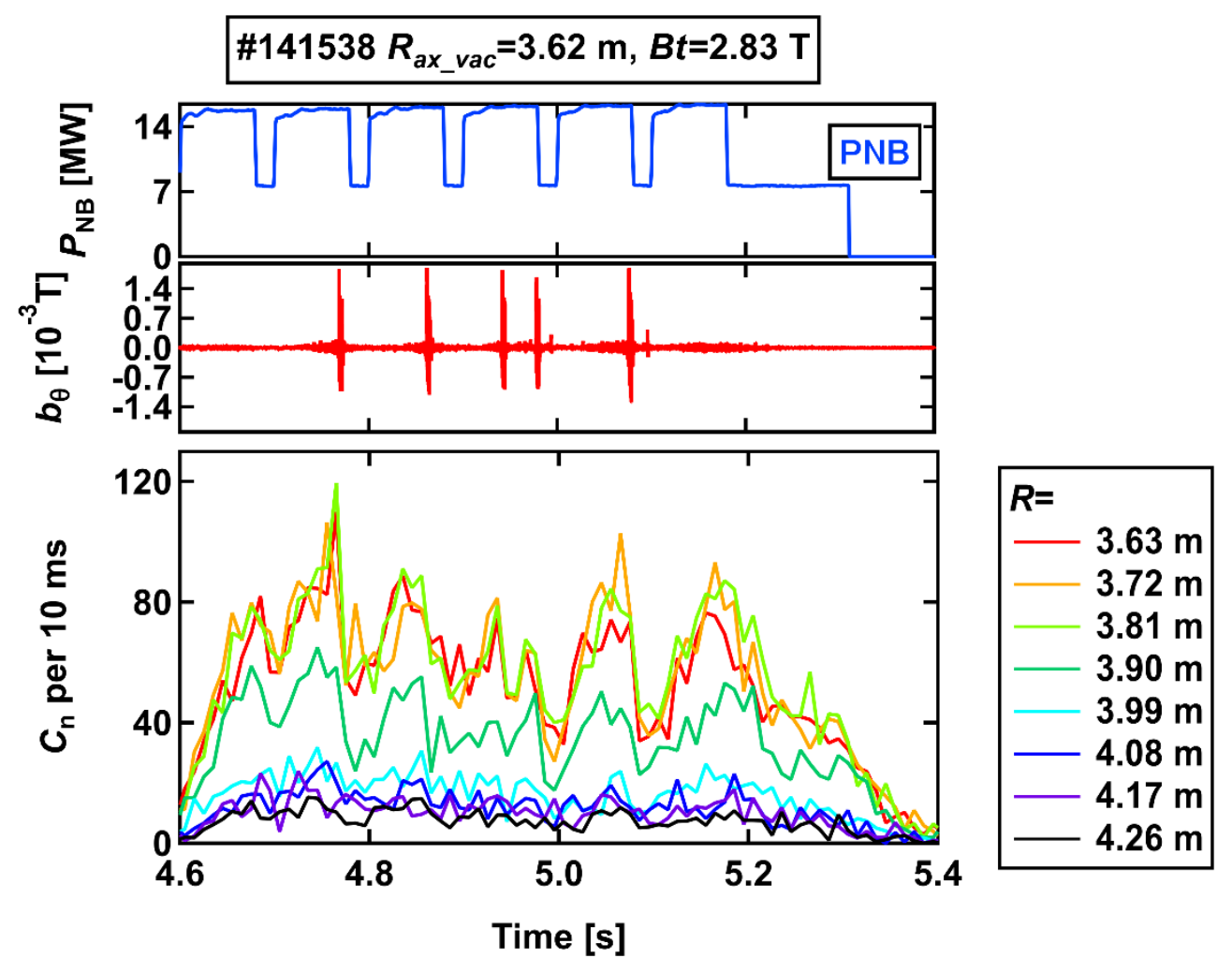

Fig. 5 Time evolutions of the injection power of PNB, magnetic fluctuation amplitude, and neutron counting rates. Here, $R$ of $3.72 \mathrm{~m}, 3.90 \mathrm{~m}$, and $4.17 \mathrm{~m}$ correspond to near $R_{\mathrm{ax}}$, normalized minor radius of 0.5 , and normalized minor radius of 1.0 , respectively. Neutron counting rate in the central channel ( $R$ of $3.63 \mathrm{~m}$ ) to the middle channel ( $R$ of $3.90 \mathrm{~m}$ ) decreases by around $50 \%$ due to EIC burst. 

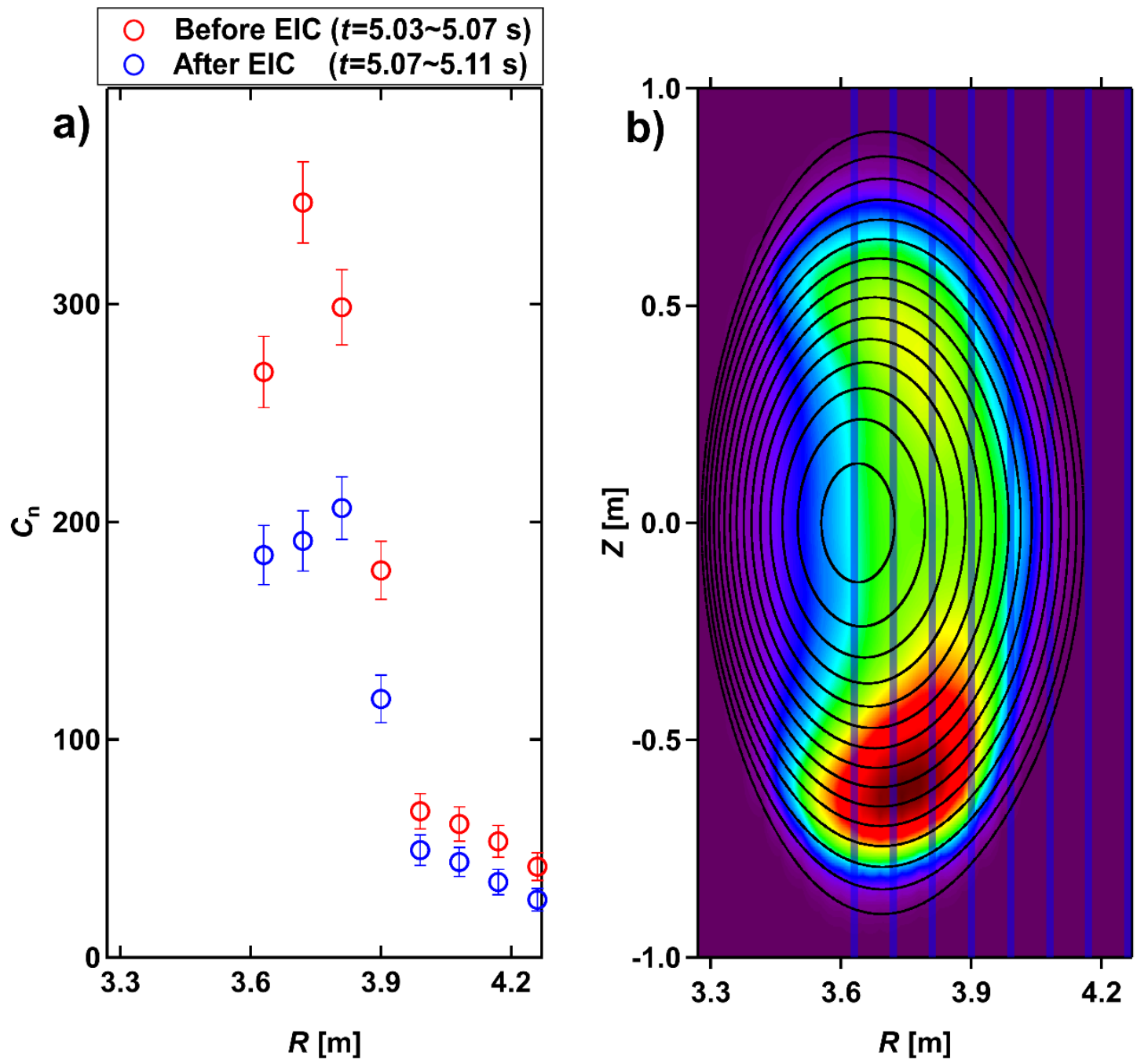

Fig.6 a) Line-integrated neutron profiles before and after EIC bursts (\#141538). Here, error bar corresponds to the statistical error. Neutron counts in core channel are significantly decreased due to EIC burst. b) A plasma shape (black dots), sight lines of VNC (blue lines), and the typical density profile of a helically trapped ion (image plot). Relatively flat line-integrated neutron profile in core channels are expected in the case of helically trapped ion compared with co-going ion. A decrease of neutron counts in central channels indicates that helically-trapped beam ions are lost due to EIC bursts. 